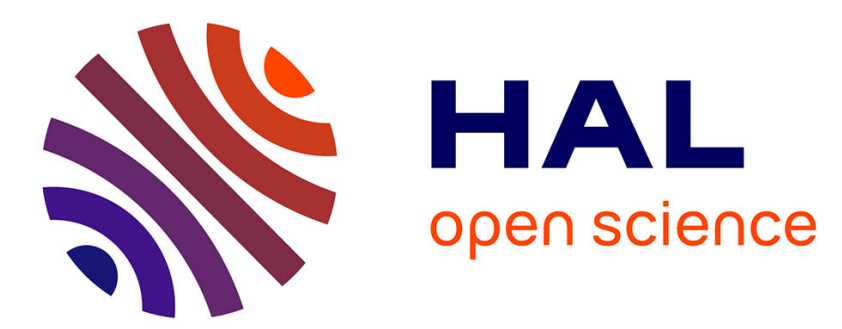

\title{
SPIN DEPENDENT NN AND N[MATH] INTERACTION FROM THE PARIS POTENTIAL
}

B. Loiseau

\section{To cite this version:}

B. Loiseau. SPIN DEPENDENT NN AND N[MATH] INTERACTION FROM THE PARIS POTENTIAL. International Symposium on High Energy Spin Physics 6, 1984, Marseille, France. pp.C2-339C2-351, 10.1051/jphyscol:1985240 . jpa-00224554

\section{HAL Id: jpa-00224554 https://hal.science/jpa-00224554}

Submitted on 1 Jan 1985

HAL is a multi-disciplinary open access archive for the deposit and dissemination of scientific research documents, whether they are published or not. The documents may come from teaching and research institutions in France or abroad, or from public or private research centers.
L'archive ouverte pluridisciplinaire HAL, est destinée au dépôt et à la diffusion de documents scientifiques de niveau recherche, publiés ou non, émanant des établissements d'enseignement et de recherche français ou étrangers, des laboratoires publics ou privés. 


\title{
SPIN DEPENDENT NN AND NN INTERACTION FROM THE PARIS POTENTIAL
}

\author{
B. Loiseau \\ Division de Physique Théorique*, Institut de Physique Nuczéaire, \\ 91406 Orsay, France \\ and \\ LPTPE, Université Pierre et Marie Curie, 75230 Paris Cedex 05, France
}

Résumé - Dans cet exposé nous voulons illustrer ce qui est compris pour les interactions Nucléon-Nucléon (NN) et Nuclẻon-antinucléon (NN) - leur dépendance en spin en particulier - en termes d'échange de mésons et êventuellement de résonances pion-Nucléon ( $\pi N)$ à 1 'aide de l'exemple du Potentiel de Paris.

Abstract - In this talk we would like to illustrate what is understood in low and medium energy NN and $N \bar{N}$ interactions - their spin dependence in particular - in terms of meson exchanges and eventually $\pi \mathrm{N}$ resonances through the example of the Paris potential.

\section{I - INTRODUCTION}

In the experimental study of the NN interaction at low and medium energy it was early realized / $/ /$ that the observed non-zero values for NN polarization and depolarization parameters were a signal to the existence of the spin dependence of the NN interaction. Besides a central force not only a long ranged tensor but also a medium ranged spin-orbit force were necessary. After the discovery of the $\pi$-meson, postulated by Yukawa /2/ as being the mediator of the NN force, one has found that the long ranged one-pion-exchange (OPE) force, which is of spin-spin and tensor type, did not generate the needed amount of spin-orbit force. Such a component was then thought to be generated by $2 \pi$-exchange, in particular $2 \pi$ correlated in $P$-wave $i . e$. the vector-isovector $\rho$-meson ( $760 \mathrm{MeV}$ ) considered in the one-boson-exchange (OBE) models. The relatively large size of the differential cross section, $\mathrm{d} \sigma / \mathrm{d} \Omega$, indicates an intermediate range attraction not explained by the pion exchange but again by $2 \pi$ exchange, specially correlated in S-wave (the non-observed scalar-isoscalar " $\varepsilon$ " ( $2550 \mathrm{MeV}$ ) introduced in $O B E$ potential models). When the existence of the excited states of the Nucleon was demonstrated in the $\pi \mathrm{N}$ scattering experiments, mainly that of the spin $3 / 2$, isospin $3 / 2, \Delta(1232 \mathrm{MeV})$, it became evident that any theoretical approach to the low and intermediate energy $\mathrm{NN}$ interaction should take into account this supplementary degree of freedom. The $2 \pi$-exchange box diagram with a nucleon and a $\Delta$ and that with two- $\Delta$ intermediate states contribute to the medium range attraction and to the nonlocality (energy dependence in particular) of the NN force. Part of the short range repulsion has been then accounted for by the $\omega(783 \mathrm{MeV})$-exchange. Furthermore the finite size of the nucleon and its structure in term of quarks, proven in deep elastic scattering experiments, lead to think that the short range NN force arising from quark interactions (quark-gluon exchanges) is of complicated nature.

The most appropriate tool to derive the long and medium ranged NN force taking into account the pionic and isobaric degree of freedom is that of the use of dispersion relations /3/. After the pioneering works of ref. (4), there has been several attempts trying to explain and reproduce the complexity of the NN interaction in these terms $15,6,7 /$. In this talk, to illustrate what is theoretically known on the long and intermediate range NN force at medium energy, we shall restrict ourselves to the

\footnotetext{
״Laboratoire Associé au C.N.R.S.
} 
approach to the problem by the Paris group /7/. It is not the only group which has studied this, in particular there has been a large amount of work performed by the Stony-Brook group $/ 6 /$.

This talk, not being a review, we apologize to authors who have devoted some of their time to the study of the $\mathrm{NN} / \overline{\mathrm{N}}$ interaction and who will not be quoted here. We refer, for completness, to the recent $N N$ and $N \bar{N}$ reviews of ref. (8) and (9) respectively.

After a brief recall in section II of how was built the Paris NN potential we shall illustrate, in section III, the spin dependence of the NN interaction by summarising the effect of the different components of the force on different NN observables at low energy $1 . e$. for $\mathrm{T}_{\mathrm{L}}$ (1aboratory kinetic energy) less than $.35 \mathrm{GeV}$ or $\mathrm{p}_{\mathrm{L}} \leqslant .88 \mathrm{GeV} / \mathrm{c}$. Section IV will demonstrate how one can use the dispersion relation approach to derive theoretical constraints on the NN inelasticity parameters in the one- and two pion production region, $.35 \mathrm{GeV} \leqslant \mathrm{T}_{\mathrm{I}} \leqslant 2.5 \mathrm{GeV}$ or $.88 \mathrm{GeV} / \mathrm{c} \leqslant \mathrm{p}_{\mathrm{L}} \leqslant 3.3 \mathrm{GeV} / \mathrm{c}$. Section $V$ will illustrate the spin dependence of the Paris NN interaction built up from constrained phenomenology and through $G$ parity transformation from the Paris Potential. Some concluding remarks and outlook will be drawn in the last section.

\section{II - RECALL ON THE PARIS POTENTIAL}

Let us here recall briefly some formulae showing how was built the Paris Potential /7/. The spin-isospin dependence of the NN scattering matrix can be written as

$$
M(w, t)=\sum_{i=1}^{5}\left[3 \mathrm{p}_{i}^{+}(w, t)+2 \mathrm{p}_{i}^{-}(w, t) \cdot \vec{\tau}^{1} \cdot \vec{\tau}^{2}\right] \mathrm{P}_{i}
$$

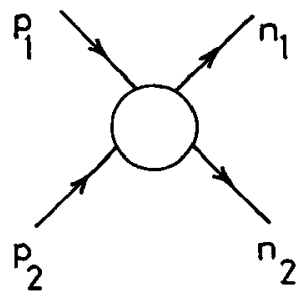

Fig. 1 - Labeliting of the nucleons where $w$ and $t$ are the usual Mandelstam variables (see Fig. 1)

$$
\begin{aligned}
& w=\left(p_{1}+p_{2}\right)^{2} \\
& t=\left(p_{1}-n_{1}\right)^{2}
\end{aligned}
$$

and $P_{i}$ the 5 following perturbative invariants,

$$
\begin{gathered}
{\left[\mathrm{P}_{1}=1^{1} 1^{2}, \mathrm{P}_{2}=\gamma^{2} \cdot \mathrm{N}_{1} 1^{1}+1^{2} \gamma^{1} \cdot \mathrm{N}_{2}, \quad \mathrm{P}_{3}=\gamma^{2} \cdot \mathrm{N}_{1} \gamma^{1} \cdot \mathrm{N}_{2}, \quad \mathrm{P}_{4}=-\gamma^{2} \cdot \gamma^{1},\right.} \\
\left.\mathrm{P}_{5}=\gamma_{5}^{2} \gamma_{5}^{1}\right]
\end{gathered}
$$

The $\tau^{i}$ and $\gamma^{i}$ are the isotopic spin Pauli and Dirac matrices of the nucleon $i(i=1,2)$ respectively and

$$
N_{1}=\frac{1}{2}\left(p_{i}+n_{i}\right), \quad I=1,2
$$

The long range OPE amplitude (pion pole) with pseudoscalar coupling is then

$$
\overrightarrow{P_{5}}=-\frac{1}{2} \frac{g_{\pi N}^{2}}{-t+\mu^{2}}
$$

where $g_{\pi N}$ is the $\pi N$ coupling constant, $g_{\pi N / 4 \pi}^{2}=14.43$ and $\mu$ the pion mass.

The intermediate range $2 \pi$-exchange amplitude is calculated with the use of disper- 
sion relations and unitarity. One can basically write

$$
M(w, t)=\frac{1}{\pi} \int_{4 \mu^{2}}^{\infty} \frac{d t^{\prime}}{t^{\prime}-t} \operatorname{Im} M\left(w, t^{\prime}\right)
$$

The unitarity of the $S$ matrix, $S S^{+}=1$, with $S=1+i M$, when saturated by $t$-channe 1 exchanges leads to

$$
\langle\mathrm{N} \overline{\mathrm{N}}|\operatorname{Im} \mathrm{M}| \mathrm{N} \overline{\mathrm{N}}\rangle=\left\langle\mathrm{N} \overline{\mathrm{N}}|\mathrm{M}| 2 \pi><2 \pi\left|\mathrm{M}^{+}\right| \mathrm{N} \overline{\mathrm{N}}\right\rangle+\left\langle\mathrm{N} \overline{\mathrm{N}}|\mathrm{M}| 3 \pi><3 \pi\left|\mathrm{M}^{+}\right| \mathrm{N} \overline{\mathrm{N}}\right\rangle+\ldots
$$

This equation gives Im $M$ in terms of the $N \bar{N}+2 \pi$ amplitude itself related to the $\pi N$ amplitude. This allows to write for the $p_{i}^{+}(w, t)$ subtracted dispersion relations of the type /7/ (we suppress the $i$ and + or - dependence for simplicity) :

$$
p(w, t)=s_{0}(t)+s_{1}(t)\left(w-w_{0}\right)+\frac{w-w_{0}}{\pi^{2}} \int_{4 \mu^{2}}^{\infty} \frac{d t^{\prime}}{t^{\prime}-t} \int_{4 m^{2}}^{\infty} \frac{d w^{\prime}-w^{\prime}}{w^{\prime}}\left[\frac{y\left(w^{\prime}, t^{\prime}\right)}{w^{\prime}-w_{0}}+w+t \bar{t}\right]
$$

$\mathrm{m}$ being the nucleon mass, $w_{0}=2 \mathrm{~m}^{2}-t / 2$ the subtraction point and $\overline{\mathrm{t}}=4 \mathrm{~m}^{2}-\mathrm{w}-\mathrm{t}$.

The $S_{0}(t)$ and $S_{1}(t)$ subtractions correspond to the exchange of $2 \pi$ correlated in a $S-$ and $P-$ wave respectively and are calculated in terms of the $N \bar{N} \rightarrow 2 \pi \quad S-$ and $P-$ wave helicity amplitudes /10/. The $\mathrm{S}$ - wave exchange, responsable of the needed medium range attraction, is replaced in the $O B E$ models by a fictitious scalar, isoscalar $E$ or $\sigma$ meson. The $P-$ wave exchange, replaced by the vector, isovector $\rho(770 \mathrm{MeV})$ meson in $\mathrm{OBE}$ models, is the agent of the medium range spin-orbit force and it also diminishes the strong tensor force arising from the $\pi$-exchange. The double spectral functions $y\left(w^{\prime}, t^{\prime}\right)$ are calculated in terms of the absorptive parts $\operatorname{Im} A^{\pi N}$ and $\operatorname{Im} B^{\pi N}$ of the $\pi N$ scattering amplitude as

$$
y\left(w^{\prime}, t^{\prime}\right)=\int_{(m+\mu)^{2}}^{s^{\prime}} d s^{\prime} \int_{(m+\mu)^{2}}^{s^{\prime \prime}} d s^{\prime \prime} K\left(w^{\prime}, t^{\prime}, s^{\prime}, s^{\prime \prime}\right) \operatorname{Im} A^{\pi N}\left(s^{\prime}, t^{\prime}\right) \operatorname{Im} B^{\pi N}\left(s^{\prime \prime}, t^{\prime}\right)
$$

$s^{\prime}$ and $s^{\prime \prime}$ being the $\pi N$ sub energies (see Fig. 2). The term with w replaced by $\bar{t}$ correspond to the crossed diagram (Fig. $2 b) . K\left(w^{\prime}, t^{\prime}, s^{\prime}, s^{\prime \prime}\right)$ is the Mandelstam Kernel which defines the domain of integration ( $s^{\prime}{ }_{\max }$ and $s^{\prime \prime}{ }_{\max }$ ).

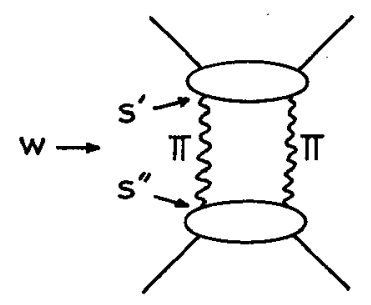

a)

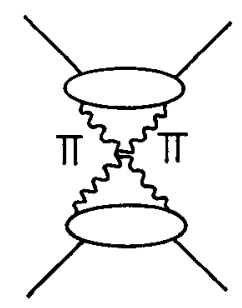

b)

Fig. 2 - Diagrams representing the uncorrelated $2 \pi$ exchanges. The blobs represent the $\pi N$ interaction with sub energies $s^{\prime}$ and $s^{\prime \prime}$, Including the nucleon pole term.

The $\pi N$ absorptive parts are known from phase shift analysis (PSA) in terms of partial wave amplitudes as

$$
\operatorname{Im} A^{\pi N}, B^{\pi N}(s, t)=\sum_{\ell}(2 \ell+1) \operatorname{Im} T_{\ell}^{\pi N}(s) P_{\ell}\left(1+\frac{t}{2 q^{2}(s)}\right)
$$


In the Paris potential use was made of the Glasgow 70, $\pi N-$ PSA /11/.These should be replaced by more up to date $\pi N-P S A$ as that of Karlsruhe-Helsinki 78, /12/, although the dominant effect, as it will be show later (see section IV), arises from the well known $\pi N, \ell=1$, spin-isospin $3 / 2$ wave i.e. the $\Delta$ resonance which dominates the low energy $\pi \mathrm{N}$ scattering.

At low energy and when the one-pion production is still small $i . e$. for $T_{1} \leqslant 3.5 \mathrm{GeV}$ the use of the Schrödinger equation with a NN potential is very adequate to describe the NN interaction. The NN amplitudes previously described lead in configuration space and for a given isospin $I=0$ or 1 , to a potential of the type :

$$
v^{I}=v_{C}^{I}+v_{S S}^{I}+v_{T}^{I}+v_{L S}^{I}+v_{S O 2}^{I}
$$

with the following centra1, spin-spin, tensor, spin-orbit and quadratic spin-orbit terms,

$$
\begin{aligned}
& V_{C}=U_{C}(r)+\frac{p^{2}}{m} W_{C}(r)+W_{C}(r) \frac{p^{2}}{m} \\
& V_{S S}=\left[U_{S S}(r)+\frac{p^{2}}{m} W_{S S}(r)+W_{S S}(r) \frac{p^{2}}{m}\right] \vec{\sigma}^{1} \cdot \vec{\sigma}^{2} \\
& V_{T}=U_{T}(r)\left[3 \frac{\vec{\sigma}_{T}^{1} \cdot \vec{r}_{\sigma^{2} \cdot \vec{r}}}{r^{2}}-\vec{\sigma}^{1} \cdot \vec{\sigma}^{2}\right]=U_{T}(r) S_{12}(\vec{r}) \\
& V_{L S}=U_{L S}(r) \vec{L} \cdot \vec{S}=\frac{1}{2} U_{I S}(r) \vec{L} \cdot\left(\vec{\sigma}^{1}+\vec{\sigma}^{2}\right) \\
& V_{S 02}=U_{S 02}(r) \frac{1}{2}\left[\vec{\sigma}^{1} \cdot \vec{L}_{L^{2}} \vec{\sigma}^{2} \cdot \vec{L}+\vec{\sigma}^{2} \cdot \vec{L} \vec{\sigma}^{1} \cdot \vec{L}\right]
\end{aligned}
$$

In eq.(2.12) the isospin superscripts have been dropped out for simplicity. It can be seen that the non-locality has been cast into a velocity dependent term in the central and spin-spin components. The phenomenological short range part $r \leqslant .8$ fm added to the theoretical long and medium range part arising from one-pion-, two-pionexchanges, as described above and $\omega$-and $A_{1}$-exchanges, as part of the $3 \pi$-exchanges, is then fitted in such a way that the full potential reproduces well the NN data /13/.

\section{III - SPIN DEPENDENCE OF THE NN INTERACTION}

The study of the correlations between the spin components of the Paris Potential and NN observables has been fully described in ref. (14) to which we refer the interested reader for details. We here give a summary of the main conclusions. The velocity dependent component, attractive at low energy and repulsive at high energy plays a role at all energies. The polarisation $P$, the depolarisation $D$ and the parameters $D_{t}$, $A, R, C_{K P}$ and $C_{N N}$ are good tests for the tensor, spin orbit and, to a smaller extent, quadratic spin-orbit forces. The isovector tensor force contribution is important at low energy and that of the isovector spin-orbit at high energy. The isoscalar tensor force effect is large at all energies and that of the isoscalar spin-orbit force rather small. The potential without quadratic spin-orbit term reproduces well the experimental data for $\mathrm{T}_{\mathbf{L}} \leqslant .15 \mathrm{GeV}$.

IV - THEORETICAL CONSTRATNTS ON NN INELASTICITIES UP TO $T_{L}=2.5 \mathrm{GeV}$ OR $\mathrm{P}=3.3 \mathrm{GeV} / \mathrm{c}$

We show here how theoretical constraints on high partial wave NN inelasticities can be obtained using the dispersion relation approach. Let us recall that, if the partial wave of the $S$ matrix are parametrized for uncoupled states as

$$
s_{J}=\eta_{J} e^{2 i \delta} J
$$


and for coupled states as

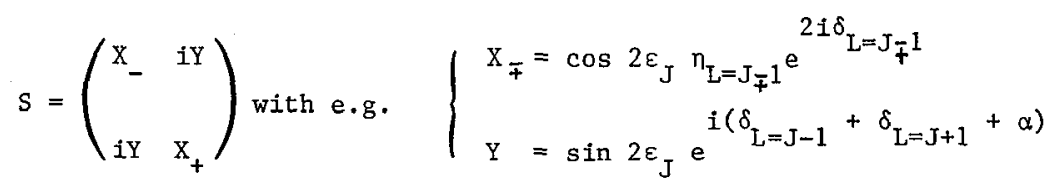

the total inelastic cross section is

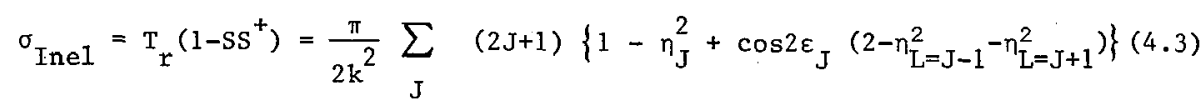

The coefficient $2 J+1$ enhances the peripheral partial wave (large J) contribution to $\sigma_{\text {Inel }}$. On the other hand, the use of unitarity tell us

$$
\sigma_{\text {Ine1 }}=\sum_{\begin{array}{c}
\beta \\
\text { Inelastic } \\
\text { channel }
\end{array}}|\langle\mathrm{NN}|\mathrm{T}| \beta\rangle|^{2}=\operatorname{Im}\left\langle\mathrm{NN}\left|\mathrm{T}^{\text {Ine1 }}\right| \mathrm{NN}\right\rangle
$$

and for $.4 \mathrm{GeV} \leqslant \mathrm{T}_{\mathrm{L}} \leqslant 1 \mathrm{GeV}, \beta=\mathrm{NN} \pi$ and $\pi d$ and for $1 \mathrm{GeV}<\mathrm{T}_{\mathrm{L}} \leqslant 2.5 \mathrm{GeV}, \beta=\mathrm{NN \pi}$ and $\mathrm{NN} \pi \pi$. We are not going to consider here the $\pi \mathrm{d}$ channel, the cross section of which peaks at $\mathrm{T}_{\mathrm{L}} \sim .65 \mathrm{GeV}$ with a value of $3.2 \mathrm{mb}$. The peripheral part of $\mathrm{T}^{\mathrm{Ine}}$ is given by $2 \pi$-exchange and the high partial wave contribution to $\sigma$ onel (eq. 4.4) can then be calculated from the imaginary parts of the diagrams of Inel Fig. 3

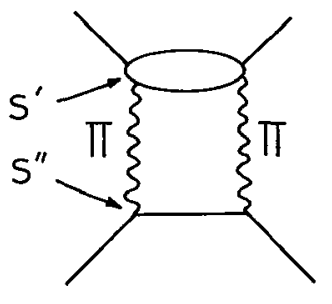

a) $1 \pi \mathrm{N}$ blob

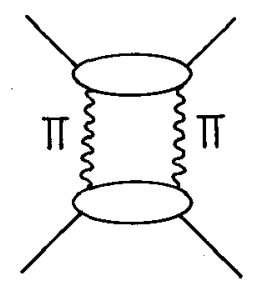

b) $2 \pi \mathrm{N}$ blobs

Fig. 3 - $2 \pi$-exchange diagrams, the imaginary parts of which contribute to $\sigma_{\text {Inel }}$. The nucleon pole is excluded from the $\pi N$ blobs.

where the blobs represent the $\pi \mathbb{N}$ interaction without the nucleon-pole contribution. One obtains (see eq. 2.8)

$$
\operatorname{Im} T^{\operatorname{Ine} 1}(w, t)=\frac{1}{\pi} \int_{4 \mu^{2}}^{\infty} \frac{d t^{\prime}}{t^{\prime}-t} y\left(w, t^{\prime}\right)
$$

The double spectral functions $y\left(w, t^{\prime}\right)$ are given by eq. (2.9). Projecting eq. (4.5) on partial wave, one obtains for the inelasticity parameters in Born approximation

$$
\eta_{J}^{2}=1-\operatorname{Im} h_{J}\left(k^{2}\right)
$$

with

$$
\operatorname{Im} h_{J}\left(k^{2}\right)=\int_{4 \mu^{2}}^{\infty} d t^{\prime} Q_{J}\left(1+\frac{t^{\prime}}{2 k^{2}}\right) y\left(w, t^{\prime}\right)
$$


For a given energy, $w=4\left(m^{2}+k^{2}\right)$, and a given $J$, the Legendre function of the second kind $Q_{J}$ acts like a damping factor at high $t^{\prime}$, suppressing the unknown short range, heavy meson exchanges or quarks forces, contributions to $y\left(w, t^{\prime}\right)$. Nevertheless depending on $J$ and on the energy our results will show some sensitivity to the maximum value of $t^{\prime}$, $t_{\text {max }}^{\prime}$, up to which we shall perform the integration.

Following ref. (16) one can also unitarize via a K-matrix procedure. One writes the full partial wave $S-m a t r i x$ as

$$
S_{J}=\frac{1+i K_{J}}{1-i K_{J}}
$$

the real $\mathrm{K}_{\mathrm{J}}$ matrix being

$$
\mathrm{K}_{J}=\mathrm{K}_{\mathrm{J}}^{+}=\left(\begin{array}{cc}
\mathrm{K}(\mathrm{J}) & \mathrm{K}(\mathrm{J}) \\
\mathrm{NN} \rightarrow \mathrm{NN} & \mathrm{NN} \rightarrow \beta \\
\mathrm{K}(\mathrm{J}) & \mathrm{K}(\mathrm{J}) \\
\beta \rightarrow \mathrm{NN} & \beta \rightarrow \beta
\end{array}\right)
$$

If one neglects the $K_{\beta} \rightarrow \beta$ transitions one can show that

$$
\underset{N N \rightarrow N N}{S(J)}=\frac{1+i \tilde{K}_{N N}(J)}{1-i \tilde{K}_{N N}(J)}
$$

with

$$
\tilde{\mathrm{K}}_{\mathrm{NN}}(\mathrm{J})=\mathrm{K}_{\mathrm{NN}} \rightarrow \mathrm{NN}(\mathrm{J})+i \operatorname{Im} \mathrm{h}_{\mathrm{J}}
$$

The $\mathrm{K}_{\mathrm{NN}} \rightarrow \mathrm{NN}(\mathrm{J})$ being related to the NN phases of the Paris potential extrapolated at high energy, e.g. for uncoupled waves we have

$$
K_{N N}(J)=\operatorname{tg} \delta_{J}
$$

The imaginary part $\operatorname{Im} h_{J}$ (see eq. 4.7) is then calculated from the 1-and 2- $\pi N$ blob diagrams (Fig. 3).

We here considered only the $S(l=0)$ and $P(l=1)$ wave of the Karlsruhe-Helsinki 78 analysis /12/. We used for the 1-blob diagram a $t$ ' cut-off of $40 \mu^{2}$. Such a value was chosen here to simulate the cancellation of the $\pi$ exchange by that of the $p$ which was demonstrated in the work of ref. (16) and also to reproduce $\sigma$ pp around $1 \mathrm{GeV} / 17 /$. The $t^{\prime} \max$ cut off for the 2-blob diagram was chosen to be $70 \mu^{2}$ in such a way that $\sigma_{\text {Inel }}^{\mathrm{pp}}$ be closed to the experimental value at $1.5 \mathrm{GeV} / 18 /$.

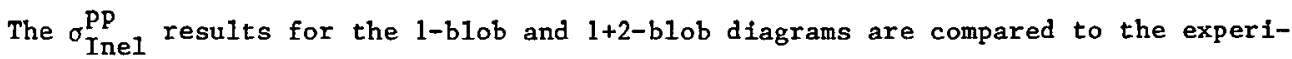
mental points $/ 17,18 /$ and to the fit of ref. (19) in Fig. 4. It can be seen that below $1 \mathrm{GeV}$ the $2-\mathrm{blob}$ contribution is negliglble. We found that the $\mathrm{P}_{33} \pi \mathrm{N}$ resonance $(\Delta, 1232 \mathrm{MeV})$ dominates the $1-$ and $2-$ blob diagrams. The contribution of each partial wave is shown in Fig. 5 in such a way that the result of progressively summing waves with successively decreasing $L$ or $J$ values is immediatly visible. The $L \geqslant 5$ waves are not very sensitive to the unitarization and to the cut off $t_{\max }$. The sensitivity of the $2 \leqslant L<5$ waves increases with energy. The lowest waves $L \leqslant 1$ which cannot be computed in a reliable way in our model give a relatively small contribution. one should notice the important contribution from $\mathrm{I}_{2}, 3 \mathrm{~F}_{3}, 1_{\mathrm{G}_{4}}, 3 \mathrm{H}_{5}$ and $3 \mathrm{~F}_{4}$. 


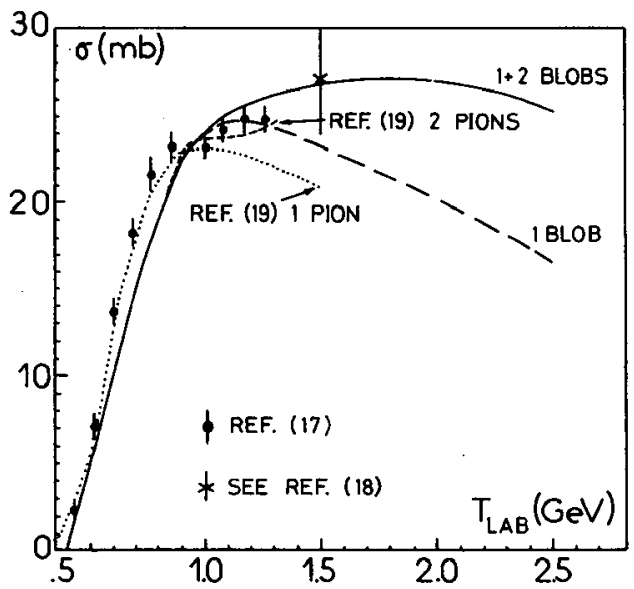

Fig. 4 - Inelastic pp cross-section from the 1 - and $1+2$ - blob diagrams

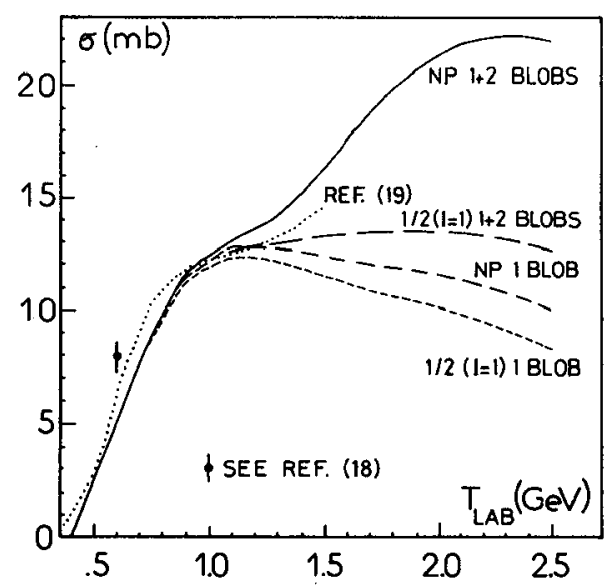

Fig. 6 - Inelastic np cross-section from the 1- and $1+2$ - blob diagrams

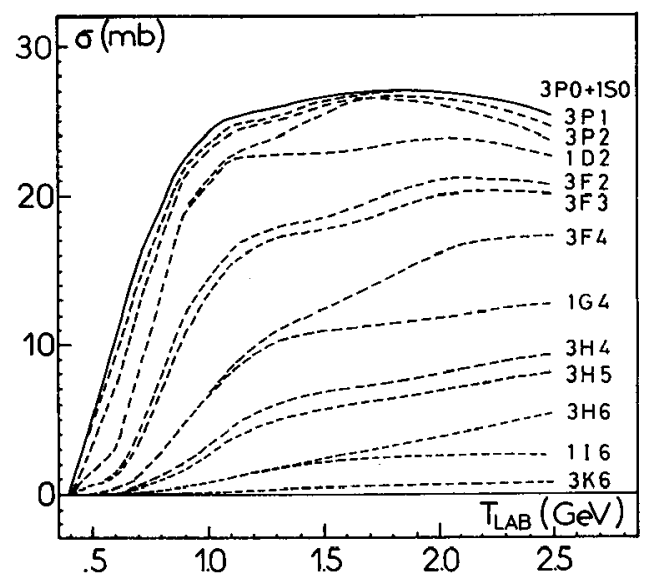

Fig. 5 - Partial wave contributions to the inelastic pp cross-section

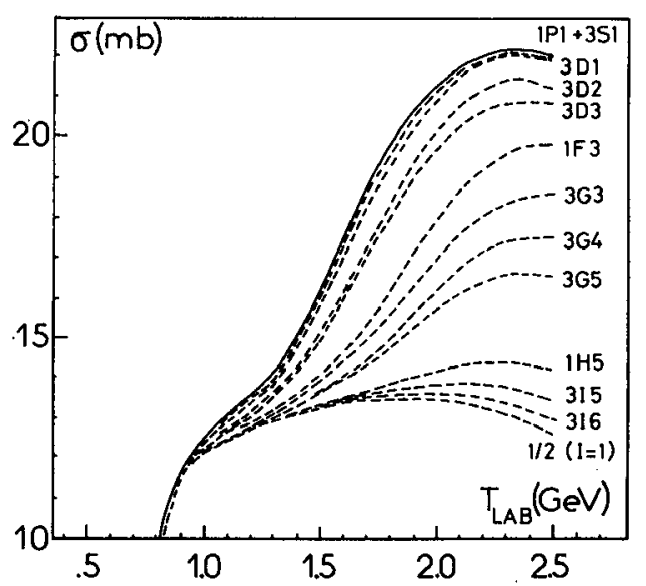

Fig. 7 - Isospin 0 partial wave contributions to the inelastic np cross-section

Similar curves for $\sigma_{\text {Inel }}^{\mathrm{np}}$ are plotted in Figs. 6 and 7 . Recall

$$
\sigma_{\text {Ine1 }}^{\text {np }}=\frac{1}{2}\left[\sigma_{\text {Ine1 }}(I=1)+\sigma_{\text {Ine1 }}(I=0)\right]
$$

As expected from the fact that the 1- blob diagram with the $\mathrm{P}_{33}$ does not contribute to the isospin $I=0$ cross-section this cross-section is very small below 1 GeV. Above $1 \mathrm{GeV}$ the 1- blob contribution to the $I=0$ crossmection arises mainly from the 1blob diagram with the $S$ and $P$, isospin $1 / 2, \pi N$ waves, the 2-blob contribution which is large comes here also mainly from $2 \mathrm{P}_{33}(2 \Delta)$ exchanges. Here (Fig. 7 ) there are important contributions to $\sigma_{\text {Ine1 }}^{\text {np }}$ from the ${ }^{3} G_{5}$ and ${ }^{3} D_{3}$ inelasticities. It should be 
noted that the 1 blob diagram with the $P_{11}$ contributes weakly to all inelasticities except to the ${ }^{3} G_{3}$ one to which it is the main contribution. Let us remark that, to our knowledge, this is the first work to use dispersion relations to calculate high partial wave NN inelasticities. Such an approach has been however recently applied to the $\pi \mathbb{N}$ scattering case $/ 20 /$.

Before closing this section we give in ref. (21) a list of authors who have also studied the NN inelasticities from the $\pi$ and $\Delta$ (and eventually $P_{11}$ ) degrees of freedom. Furthermore Kloet and $\mathrm{Tj}$ on /22/ have recently studied the model dependence of the NN inelasticities of some of these calculations.

\section{$V$ - SPIN DEPENDENCE OF THE PARIS NN̄ INTERACTIONS}

At low energy for $\mathrm{T}_{\mathrm{L}} \leqslant .4 \mathrm{GeV}$ or $\mathrm{P}_{\mathrm{L}} \leqslant .96 \mathrm{GeV} / \mathrm{c}$ the use of the Schrödinger equation for $N \bar{N}$ is as adequate as in the $N N$ case, however one has to take into account the annihilation. One can use coupled channel equation /23/ or equivalently introduce an optical potential /24/. The Paris NN interaction /25/ uses an optical potential

$$
\mathrm{v}_{\mathrm{N} \overline{\mathrm{N}}}=\mathrm{U}_{\mathrm{N} \overline{\mathrm{N}}}-\mathrm{i} \mathrm{w}_{\mathrm{N} \overline{\mathrm{N}}}
$$

The long and intermediate range real potential $\mathrm{U}_{\mathrm{NN}}$ is given by the G-parity transform of the Paris NN potential

$$
U^{\text {theoretical }}(N \bar{N}, n \pi \text { exchange })=(-1)^{n} v^{\text {theoretical }}(N N, n \pi \text { exchange })
$$

The OPE, $\quad \omega$ and $A_{1}$ exchange potentials change their sign, one then obtains a strongly attractive potential which could give rise to bound states and resonances $/ 26 /$. The short range part, which could be in principle calculated in terms of quark forces, is determined phenomenologically in the Paris potential.

The imaginary part of the potential, $\mathrm{W}_{\mathrm{NM}}$ is related via the unitarity to the modulus square of the NN annihilation into mesons $/ 25,27 /$. It is expected to be of short range, the lowest mass of the $t$ exchange being that of $2 \mathrm{~m}$, and the range will be

$$
\mathrm{r}_{\mathrm{o}} \leqslant \frac{1}{2 \mathrm{~m}} \simeq .11 \mathrm{fm}
$$

It is also expected to be non-local and state dependent. In the Paris N $\vec{N}$ potential, $\mathrm{W}_{\mathrm{NN}}$ was parametrized, for both isospin, as

$$
\begin{aligned}
& \mathrm{W}_{\mathrm{NN}}^{\mathrm{I}}\left(\overrightarrow{\mathrm{r}}, \mathrm{T}_{\mathrm{L}}\right)={ }_{\mathrm{g}_{\mathrm{C}}}\left(1+\mathrm{f}_{\mathrm{C}} \mathrm{T}_{\mathrm{L}}\right)+\mathrm{g}_{\mathrm{SS}}\left(\mathrm{I}+\mathrm{f}_{\mathrm{SS}} \mathrm{T}_{\mathrm{L}}\right) \vec{\sigma}^{\mathrm{l}} \cdot \vec{\sigma}^{2}+\mathrm{g}_{\mathrm{T}_{12}} \mathrm{~S}_{12}(\overrightarrow{\mathrm{r}}) \\
& \left.+g_{L S} \frac{\vec{L} \cdot \vec{S}}{4 m^{2}} \frac{1}{r} \frac{d}{d r}\right] \frac{K_{o}(2 m r)}{r}
\end{aligned}
$$

The modified Bessel function $\mathrm{K}(2 \mathrm{mr})$ arises from the calculation of the imaginary part of a box diagram with $2 \pi^{\circ}$ and $a \mathrm{~N}$ and $\overline{\mathrm{N}}$ exchanges. The unknown short range part of $\mathrm{U}_{\mathrm{N} \overline{\mathrm{N}}}$ and the parameters $\mathrm{g}_{\mathrm{C}}, \mathrm{f}_{\mathrm{C}}, \mathrm{g}_{\mathrm{SS}}$ etc... were adjusted to fit the existing data of $\frac{d \sigma^{e l}}{d \Omega}(\bar{p} \bar{p}), \sigma^{\text {tot }}(\bar{p} \bar{p}), \frac{d \sigma^{p \bar{p}} \rightarrow n \bar{n}}{d \Omega}, \sigma^{p \bar{p} \rightarrow n \bar{n}}$ and $P(p \bar{p}), 125 /$.

Before illustrating the spin dependence, some predictions of this model on $\mathrm{d} \sigma / \mathrm{d} \Omega$ of $\bar{p} \bar{p} \rightarrow \bar{n}$ are shown in Fig. 3 of ref. (28) for $78.8 \mathrm{MeV} \leqslant T_{L} \leqslant 282.2 \mathrm{MeV}$. The predictions are rather good except at forward angle where the dip is too pronounced. This could be due to the fact that the Coulomb interaction in the entrance channel was not taken into account. Work to include this correction is in progress. Fig. 8 shows the fair agreement of the pure $I=1, \overline{p n} \rightarrow \overline{p n}, d \sigma / d t$ of the Paris potential to the values of Caro et al. $/ 29 /$ at $\mathrm{T}_{\mathrm{L}}=390 \mathrm{MeV}\left(\mathrm{p}_{\mathrm{L}}=.940 \mathrm{GeV} / \mathrm{c}\right)$. 


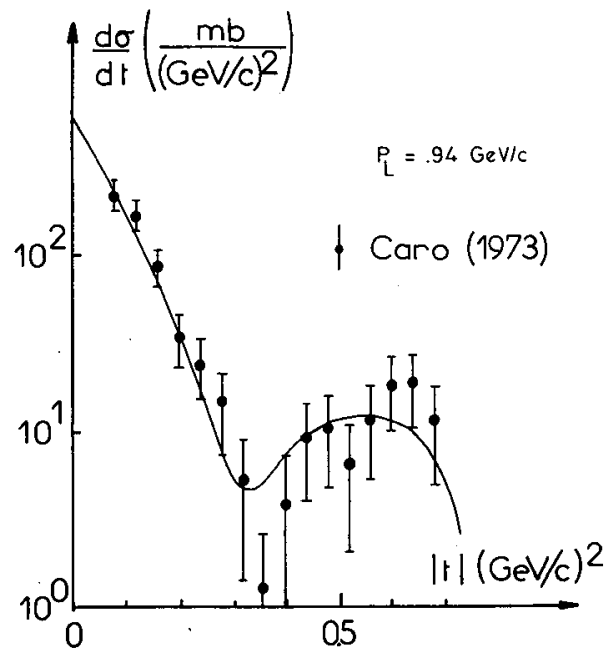

Fig. 8 - Prediction of the Paris NN̄ potential for $\mathrm{d} \sigma / \mathrm{dt}$ of $\overline{\mathrm{p}} \mathrm{h} \rightarrow \overline{\mathrm{p}}$ at $\mathrm{P}_{\mathrm{L}}=.94 \mathrm{GeV} / \mathrm{c}$ $\left(\mathrm{T}_{\mathrm{L}}=.39 \mathrm{GeV}\right)$.
The sensitivity of some $N \bar{N}$ observables to different spin components of $U_{N \bar{N}}$ and $W_{N \bar{N}}$ and to the energy dependence of $\mathrm{W} N \overline{\mathrm{N}}$ are illustrated in Figs. 9 to 11. NN Fig. 9 shows that the backward $\mathrm{d} \sigma / \mathrm{d} \Omega$, which compares well to the measurements of ref. (30), is very sensitive to the tensor component $\mathrm{U}_{\mathrm{T}}$ of $\mathrm{U}_{\mathrm{NN}}$ and to a lesser extent to that of the spin-orbit $U_{\mathrm{IS}}$. It is also very sensitive to the energy dependence of $W_{N} \bar{N}$ but much less sensitive to the sum, $W_{N \bar{N}}^{L S}+W_{N \bar{N}}^{T}$, of the spin-orbit and tensor potential of $W_{N} \bar{N}$. This strong energy dependence is expected as the presence of an imaginary part of $\mathrm{V}_{\mathrm{N}} \overline{\mathrm{N}}$ replaces a coupled channel problem where the aperture of new annihilation channels is energy dependent. This study on the polarisation at $\mathrm{T}_{\mathrm{L}}=230 \mathrm{MeV}$, compared to the measurement of ref. (31) in Fig. 10, shows a similar patern. It can be furthermore seen that below $90^{\circ}$, the polarisation is mainly given by the

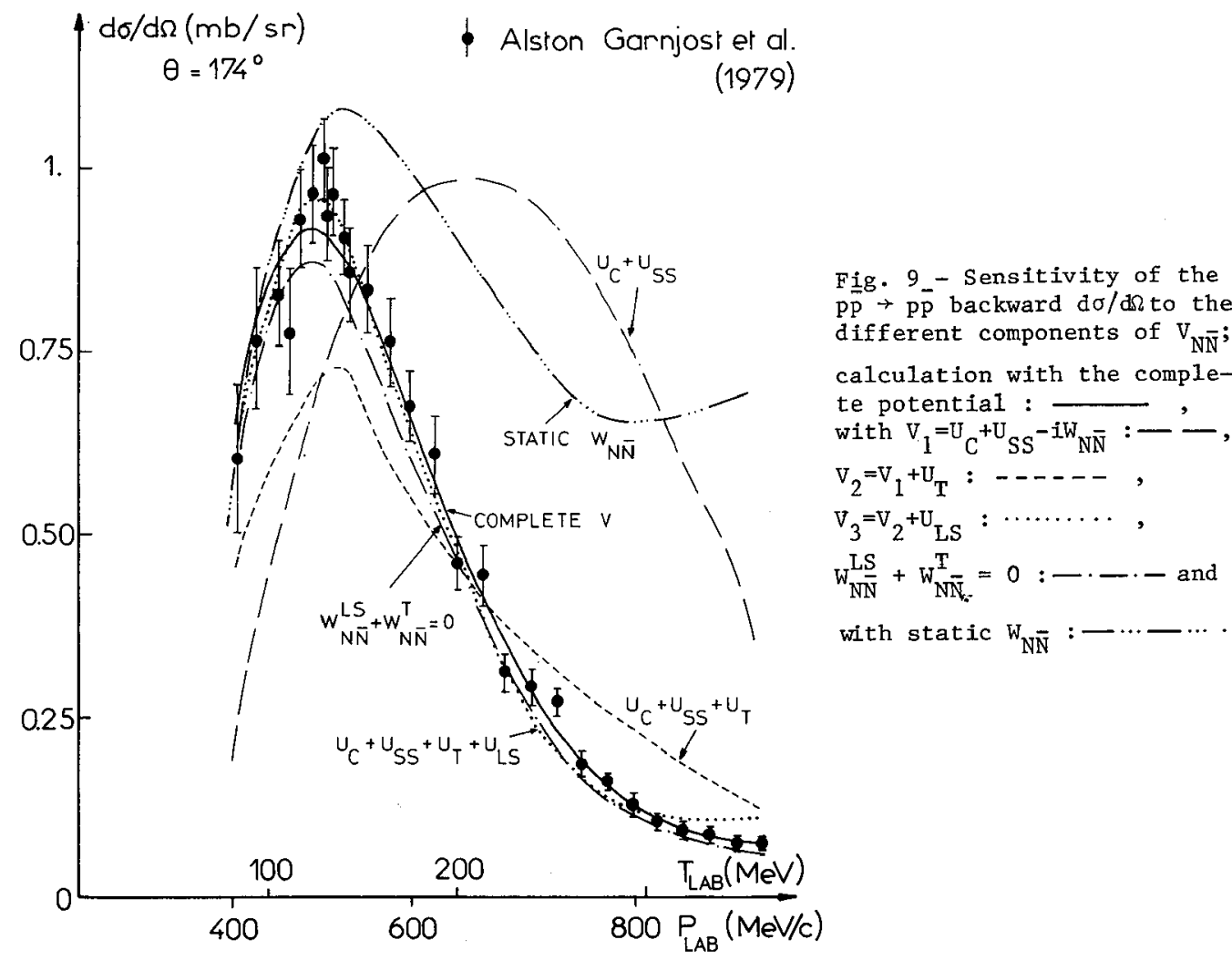




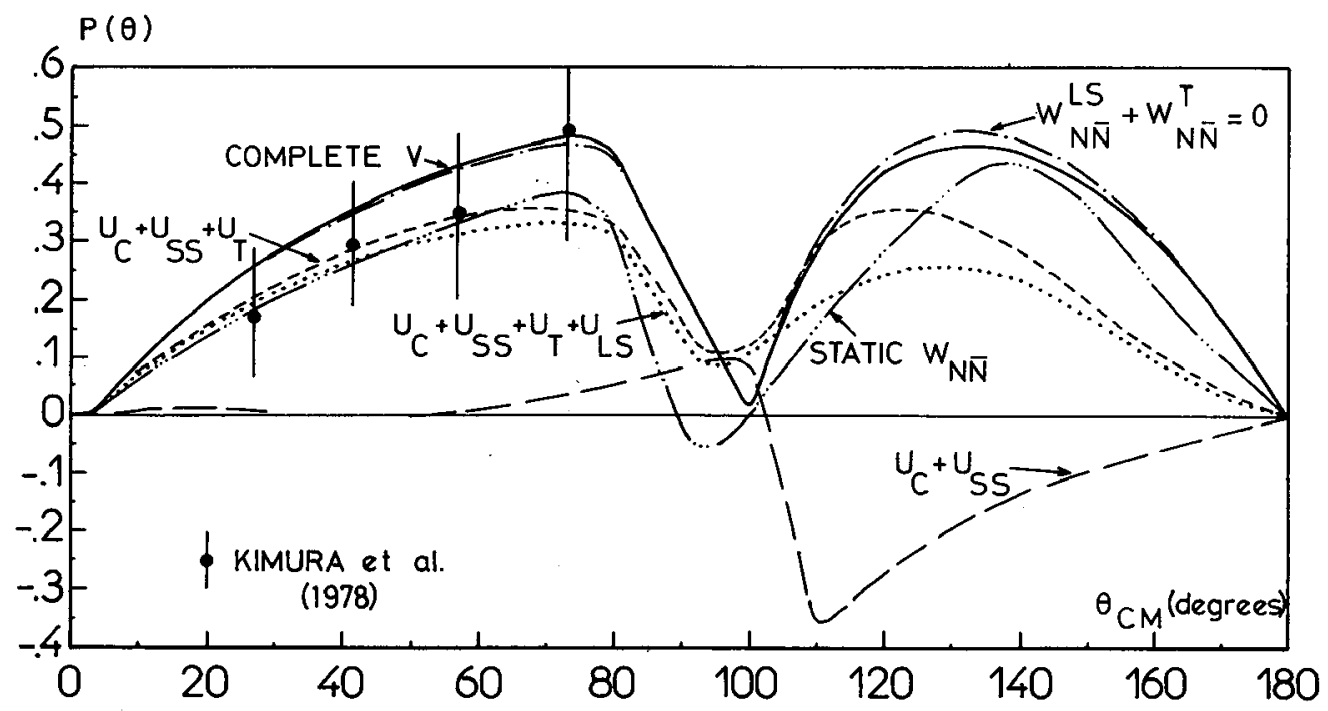

Fig. 10 - The same as in Fig. 9 but for the $\bar{p}$ Polarisation at $T_{L}=.230 \mathrm{GeV}$.

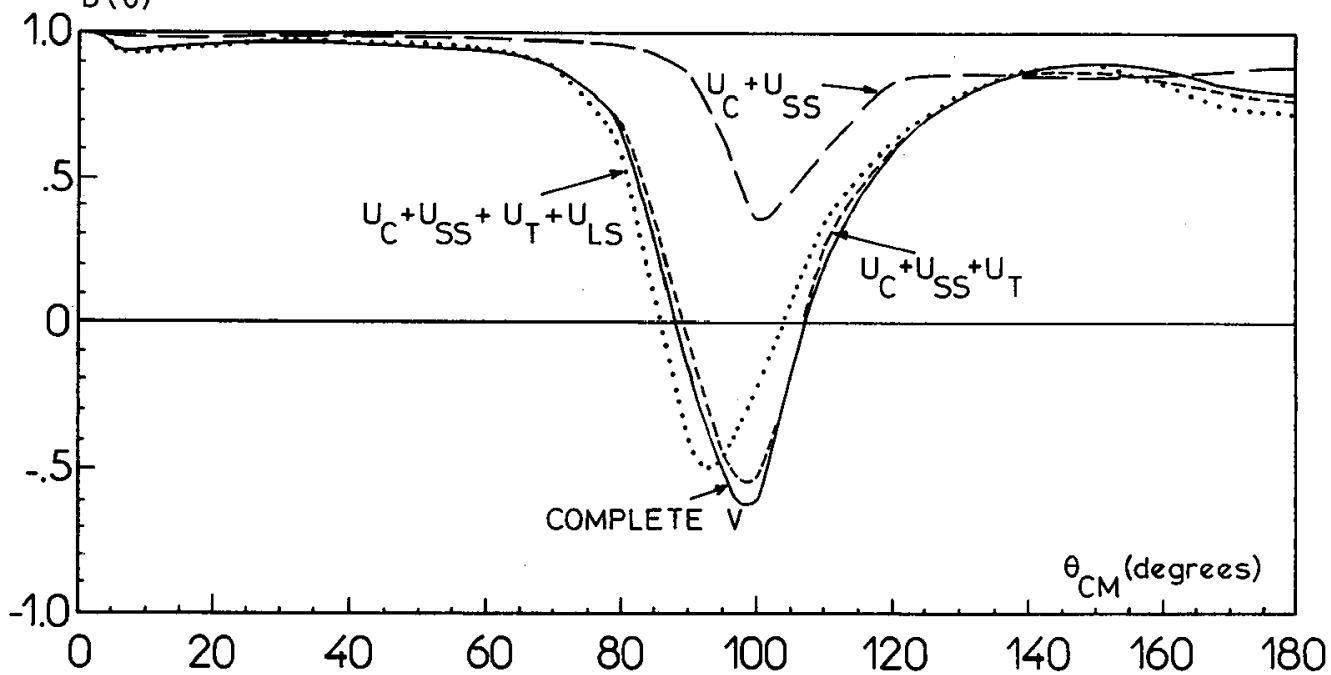

Fig. II - As in Fig. 9 but for the $\bar{p}$ Depolarisation at $T_{L}=.230 \mathrm{GeV}$. The results with $W_{N \bar{N}}^{L S}+W_{N \bar{N}}^{T}=0$ and static $W_{N \bar{N}}$, very close to that with the complete potential, are not plotted.

tensor force $U_{T}$. The depolarisation parameter (FIg. 11), insensitive to the energy dependence of $\mathrm{W}_{\mathrm{N} \overline{\mathrm{N}}}$ and to $\mathrm{W}_{\mathrm{N}}^{\mathrm{LS}}+\mathrm{W}_{\mathrm{N} \overline{\mathrm{N}}}^{\mathrm{T}}$, is also mainly given by the tensor force $\mathrm{U}_{\mathrm{T}}$. This important tensor component of $\mathrm{U}_{\mathrm{NN}}$ is due here to the coherent addition of the tensor 
forces of the $\pi-, 2 \pi-$ (in particular $2 \pi$ correlated in $P$ wave $1 . e$. the $\rho$ meson) and $\omega$ - exchanges. The spin-orbit force plays a less important role. This is the reverse situation of the NN case as has been recalled in section III and 1llustrated in ref. (14) and is a consequence of the G-Parity transformation. Such a fact has been also found and shown in ref. (32).

\section{VI - SOME CONCLUDING REMARKS AND OUTLOOK}

At low energy, $\mathrm{T}_{\mathrm{I}} \leqslant .35 \mathrm{GeV}$ or $\mathrm{P}_{\mathrm{T}} \leqslant .88 \mathrm{GeV} / \mathrm{c}$ the long and intermedlate range NN force is wel1 understood in terms of meson exchanges. The spin effects $(P, D, A, \ldots)$ are wel1 explained by a long range tensor force arising from $\pi-$ and $2 \pi-$ (correlated in $P$ - wave $i, e$. the $\rho$ ) exchange plus an intermediate range spin-orbit force (exchange of $2 \pi$ correlated in $\mathrm{P}-$ wave $i . e$. the $\rho$ ). In the Paris potential the short range part was treated phenomenologically, however one should be able to explain it if the theory of strong interaction was fully understood.

Some attemps to derive short range forces have been made within the Quantum Chromodynamic framework. These microscopic approaches /33,34/ depend on the quark-quark forces, the quark confinement mechanism and the wave function of overlapping nucleons and their dynamics. There has been also hybrid quark-nucleon models where an inner region of quarks joins in a smooth way to an outer region of nucleons $/ 35 /$.

It has been suggested by skyrme $/ 36 /$ that the nucleon could be a solition in the $\pi$ and $\sigma$ field. Recently Adkins, Nappi and Witten /37/ have quatized the Skyrme soliton and fitting the $N$ and $\Delta$ masses, they were able to describe the static properties of the nucleon within $30 \%$. A baryon-baryon force can then be calculated as resulting from a soliton-soliton interaction. A static NN potential has been recently derived in refs. (38) and (39) with the approximation that the field configuration for two solitons is a product of two single soliton fields. In ref. (39) it was found that this soliton-soliton interaction gives rise to a long range tensor potential very close to that of a $\pi$ exchange of zero mass. At short range a central repulsion is obtained. Furthermore not only $N N \rightarrow$ NN but also $N N \rightarrow$ N $\Delta$ and $N \Delta \rightarrow N \Delta$ static potentials were derived. Some non static force terms has been also considered in ref. (40).

Theoretical high partial wave inelasticities arising from $\pi$ and $\Delta$ degrees of freedom, should be used as constraints, with some model dependent errors, in PSA, in particular waves with $\mathrm{L} \geqslant 4$ for $\mathrm{T}_{\mathrm{L}} \leqslant 1 \mathrm{GeV}\left(\mathrm{p}_{\mathrm{L}} \leqslant 1.7 \mathrm{GeV} / \mathrm{c}\right)$ and $\mathrm{L} \geqslant 5$ for $1 \mathrm{GeV}<\mathrm{T}_{\mathrm{L}} \leqslant 2.5 \mathrm{GeV}$ $\left(1.7 \mathrm{GeV} / \mathrm{c}<\mathrm{p}_{\mathrm{L}} \leqslant 3.3 \mathrm{GeV} / \mathrm{c}\right)$.

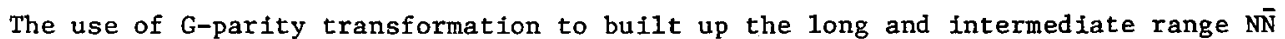
force from the NN force tests coherences between meson exchanges. The study of spin effects with the Paris $N \bar{N}$ potential has shown the importance of a strong tensor force, here the $\pi+2 \pi(\rho)+\omega$ exchange tensor forces add coherently, the role of the spin-orbit force being weaker. The phenomenological annihilation, constrained to be short ranged in the Paris potential, as predicted by meson theory, shows a weak spin dependence but a strong energy dependence. There has been different trials to derive the annihilation in terms of $\mathrm{q} \bar{q}$ forces either in terms of quark rearangement models and/or interms of annihilation diagrams /41/. It should be noted that only few polarisation measurements around $230 \mathrm{MeV}$ and $379 \mathrm{MeV}$ exist so far. More data on spin observables $\left(P, D, D_{t}, \ldots\right)$ are needed to fully explain the spin dependence of the NN interaction. Some data will be hopefully soon available from LEAR /42/.

\section{ACKNOWLEDGEMENTS}

I would like to thank Professors M. Lacombe, R. Vinh Mau and Dr. S, Morioka who greatly helped me for the preparation of this talk. 


\section{REFERENCES}

1 - B.P. Nigam, Rëv. Mod. Phys. 35 (1963) 117 ; and refs. cited therein.

2 - H. Yukawa, Proc. Phys. Math. Soc. Japan 17 (1935) 48.

3 - S. Mandelstam, Phys. Rev. 112 (1958) 1344.

4 - D. Amati, E. Leader and B. Vitale, Nuovo Cimento 17 (1960) 68 ; 18 (1960) 409; Phys. Rev. 130 (1963) 750;W.N. Cottingham, R. Vinh Mau, Phys. Rev. 130 (1963)735.

5 - S. Furuichi, Supplement Progress of Theor. Physics 39 (1967) 190.

6 - G.E. Brown, Comments Nuc1. Part. Phys. 4 (1970) 140; M. Chemtob, J.W. Durso and D.O. Riska, Nuc1. Phys. B38 (1972) $\overline{1} 41$; J.W. Durso, M. Saarela, G.E. Brown and A.D. Jackson, Nuc1. Phys. A278 (1977) 445 ; G.E. Brown and A.D. Jackson, The Nucleon-Nucleon Interaction, (North-Holland, Amsterdam, 1976).

7 - W.N. Cottingham, M. Lacombe, B. Loiseau, J.M. Richard and R. Vinh Mau, Phys. Rev. D8 (1973) 800 ; R. Vinh Mau, "The Paris N-N Potential" in Mesons in Nuclei, edited by M. Rho and D. Wilkinson (North-Holland, Amsterdam, 1979) p.179; W.N. Cottingham, "Mesons and the two-nucleon potential", Rep. Prog. Phys. 41 (1979) 1509.

8 - J.J. de Swart, W.A. van der Sanden, W. Derks, NucI. Phys. A416 (1984) 299c.

9 - C.B. Dover, Nuc1. Phys. A416 (1984) 313c.

10 - G. Höhler and E. Pietarinen, Nucl. Phys. B95 (1975) 210. E. Pietarinen, Helsinki report (1978) ; P. Gauron, Paris report, IPNO/TH 78-07 (1978) ; B. Bonnier and P. Gauron, Nuovo Cimento 57A (1980) 261 .

11 - Particle Data Group, UCRL report $N^{\circ} 20030$ (1970).

12 - G. Höhler, F. Kaisex, R. Koch and E. Pietarinen, Handbook of Pion Nucleon Scattering, Physics Data 12-1, Karlsruhe (1979).

13 - M. Lacombe, B. Loiseau, J.M. Richard, R. Vinh Mau, J. Côté, P. Pirès and R. de Tourreil, Phys. Rev. C21 (1980) 861 ; M. Lacombe, B. Loiseau, R. Vinh Mau, J. Côté, P. Pirès and R. de Tourreil, Phys. Rev. 23 (1981) 2405.

14 - F. Pauss, L. Mathelitsch, J. Côté, M. Lacombe, B. Loiseau and R. Vinh Mau, Nucl. Phys. A365 (1981) 392.

15 - M. Lacombe, B. Loiseau, S. Morioka and R. Vinh Mau, Few Body Problems in Physics Vol. II contributed papers, B. Zeitnitz (editor), Elsevier 1984, p. 151 ; Paris report, IPNO/TH 84-12; Particles and Nuclei Tenth International Conference, Book of Abstracts, Vo1. 1, Heidelberg 1984, F. Güttner, B. Pooh, G. zu Put1itz Editors, Springer-Verlag, C10.

16 - J. Côté, M. Lacombe, B. Loiseau and W.N. Cottingham, Nuc1. phys. A379 (1982) 349.

17 - F. Shimizu, Y Kubota, H. Koiso, F. Sai, S. Sakamoto and S.S. Yamanoto, Nucl. Phys. A386 (1982) 571 .

18 - J. Bystricky and F. Lehar, Physics Data 11-1, Kar1sxuhe (1978).

19 - B.J. Verwest and R.A. Arndt, Phys. Rev. C25 (1982) 1979.

20 - G. Höhler, D. Grether, M. Hutt and I. Sabba-Stefanescu, Karlsruhe report, TPK 83-24 (1983).

21 - A.M. Green, J.N. Niskanen, J. Phys. G4 (1978) 1055 ;.A.M. Green,M.E. Sainio, J. Phys. G5 (1979) 503 ; ibid G8 (1982) 1337 ; W.M. Kloet and R.R. Silbar , Nucl. Phys. A338 (1980) 281; Nucl. Phys. A338 (1980) 317 ; Nucl. Phys. A364 (1981) 346 ; Phys. Rev. Lett. 45 (1980) $97 \overline{0 ;}$ J.H. Gruben and B.J. Verwest, Phys. Rev. C28 (1983) 836.

22 - W.M. Kloet and J.A. Tjon, Rutgers University report, RU-84-26 (1984).

23 - P.H. Timmers, W.A. van der Sanden and J.J. de Swart, Phys. Rev. D29 (1984) 1928.

24 - R. Bryan and R. Phillips, Nucl. Phys. B5 (1968) 201 ; C. Dover and J.M. Richard, Phys. Rev. C21 (1980) 1466.

25 - J. Côté, M. Lacombe, B. Loiseau, B. Moussallam and R. Vinh Mau, Phys. Rev. Lett. 48 (1982) 1319 .

26 - M. Lacombe, B. Loiseau, B. Moussallam and R. Vinh Mau, Phys. Rev. C29 (1984)1800.

27 - R. Vinh Mau, "NN̄ forces and annihilation in the potential approach" in Physics at LEAR with low-energy cooled antiprotons, edited by U. Gastaldi and R. Klapish, Plenum Publishing Corporation, 1984, p. 395 ; B. Loiseau, "Potential approach to Antinucleon-Nucleon interaction", Proceeding's of the Third LAMPF II Workshop L.A.N.L., Los Alamos, N.M., 1983, LA-9933-C Vo1.1 p. 366.

28 - K. Nakamura et al., Phys. Rev. Lett. 53 (1984) 885.

29 - D.E. Caro et a1., Nucl. Phys. B52 (1973) 301.

30 - M. Alston-Garnjost et al., Phys. Rev. Lett. 43 (1979) 1901.

31 - M. Kimura et al., Nuovo Cimento 71A (1982) $4 \overline{38}$; Proceedings of the Fourth European Symposium on $N \bar{N}$ interaction (CNRS, Paris, 1979) p. 539. 
32 - C. Dover and J.M. Richard, Phys. Rev. C25 (1982) 1952.

33 - D.A. Liberman, Phys. Rev. D16 (1977) $1 \overline{542}$; C. de Tar, Phys. Rev. D17 (1978) 323 ; ibid 19 (1979) 1451 ; M. Harvey, Nuc1. Phys. A352 (1981) 301, 326 ; A. Faessler, F. Fernandez, G. Lübeck and K. Shimizu, Phys. Lett. 112B (1982) 201 ; M. Oka and K. Yazaki, Nuc1. Phys. A402 (1983) 477 ; S. Furui and A. Faessler, Nucl. Phys. A397 (1983) 413 .

34 - C.W. Wong, "Yukawa, Skyrme and QCD", Tenth International Conference on Particle and Nuclei, Heidelberg 1984, Nucl. Phys. A, to be published.

35 - E. Lomon, in Hadron Subsrtucture in Nuclear Physics, edited by W.Y.P. Hwang and M.H. Macfarlane, AIP Proceedings N¹10 (1984) 117 ; L.S. Kisslinger, ibid p. 271 ; E. Lomon, these proceedings ; P.J. Mulders, Nucl. Phys. A416 (1984)99c.

36 - T. Skyrme, Proc. Roy. Soc. London 260 (1961) 127 ; Nuc1. Phys. 31 (1962) 556.

37 - G.S. Adkins, G.R. Nappi and E. Witten, Nucl. Phys. B228 (1983) 552.

38 - A. Jackson and $V$. Pasquier, Stony Brook preprint, to be published in Nucl. Phys.

39 - R. Vinh Mau, M. Lacombe, B. Loiseau, W.N. Cottingham, P. Lisboa, IPNo/TH preprint $\mathrm{N}^{\circ} 84-66$, to be published in Phys. Lett. B.

40 - Z. Hlousek, Brown University Preprint, HET 526 (1984).

41 - M. Maruyama and T. Ueda, Phys. Lett. 124B (1983) 121 ; A.M. Green, J. Niskanen, J.M. Richard, Phys. Lett. 121B (1983) $\frac{101 .}{10}$

42 - F. Bradamante, these proceedings. 